\title{
Analysis on the Application of Zigbee-based Wireless Sensor Network in Industrial Monitoring
}

\author{
Xiaodong Zhang, Jing Yang \\ Henan Mechanical and Electrical Vocational College, Zhengzhou Henan, 451191, China
}

Key Words: Zigbee, Wireless Sensor Network, Industrial Monitoring, Specific Application

\begin{abstract}
With the development of science and technology, large-scale enterprises add internal regulation equipment and necessary real-time monitoring. However, traditional circuit the wiring has followed can not offer network support and gradually reveals the incommensurate disadvantages. Communication network in a broader category has been constructed with the network development and the microcomputer regulation peculiar to embedded architecture has been accepted. Sensor network shows the miniature characteristic, and integrates the accuracy of calculation flow and more acute perception and sensing technology. Zigbee's specific sensor network has been incorporated into wireless network. New type of network meets the new requirement of industrial monitoring. On basis of what has been discussed above, the wireless sensor network on basis of Zigbee should be analyzed for application in the industrial monitoring of normal process.
\end{abstract}

\section{Introduction}

Industrial control technology, which is developing rapidly, expands the industrial control scale of overall architecture. Under such conditions, the input industrial control consumption should be cut short in order to create favorable effects in a maximum category. In traditional industrial control, wired network consumes relatively high setting cost and subsequent operation cost, which hinders the extended industrial communication. At the same time, the indispensable internal wiring of wired network hinders the allocation of equipment and can not adjust to the industrial occasions with corrosion property and complicated wiring occasions. The network constructed by wireless sensor has more comprehensive new advantages and gradually receives more attention. Industrial monitoring on basis of Zigbee is more suitable for the normal production of remote control, acquiring real-time industrial information. On this basis, the control process will be optimized and improve the actual monitoring effects in essence.

\section{New-type wireless sensor network}

The sensor network particular to wireless architecture, which covers various internal nodes, can be called WSN. Nodes in this network are integrated to miniature sensors; multi-hop network particular to organization has been set in accordance with wired communication. Setting of such kind of new network extends the covered collection category and intensively percepts the object information of designated sections. It collects and percepts the information, and sends it to designed users. Such kind of system covers the internal nodes, nodes necessary for management, and converging nodes set by sensors. The constructed network sets self-organization and offers skip transmission one by one. The transmission process will be confronted with multiple internal nodes and converge the existing internal route through subsequent processing [1]. Network connects managing nodes in series, and relies on internal nodes in order to successfully regulate sensors and manage the created network. Under normal conditions, a certain standard of minute type cell can be adopted.

It can be seen in detail that sensor nodes can be grouped into four internal modules, namely wireless communication module, processing module, energy supply module, and ontology sensor module. Ontology sensor module verifies all the covered information of testing area, and transforms original information; processing module regulates the nodes in network and preserves the collected information; module necessary for communication is closely linked with other sensors and take 
responsibility of interchanging, sending and receiving information; module necessary for energy supply adds sufficient energy for subsequent supply.

\section{New technique peculiar to Zigbee}

\section{Original technical connotation}

Zigbee, as an emerging technology, creates network transmission with lower power consumption and shorter interval. The new-type wireless sensor network technology sets accurate frequency band for ISM, which can transmit $200 \mathrm{~kb}$ per second and extend $70 \mathrm{~m}$ transmission gap. As for the fundamental characteristics, Zigbee similar to general bluetooth technology has even simpler original advantages because wireless transmission evades long-term sleep and update does not need continuation.

For example, the presupposed normal communication of industrial equipment can adopt such kind of new-type network. Wireless sensor sets the physical layer of internal architecture and relevant MAC, and follows designed protocol standards. On this basis, it again extends the initial technological ways. Standardization covers the designed protocol at initial stage and the interface equipped for application program, paying more attention to the exploration of internal security layer and the construction of data link. In addition, network also integrates the essential physical layer, link, security layer, and higher application layer.

\section{Refined network layers}

The first layer is physical layer which follows the protocol and belongs to the downmost layout layers. Physical layer connects with the external and takes charge of daily network transmission. It is able to regulate data, send data and receive transmitted information.

The second layer is link layer which is also named data link. Data link sets up checkable standards including logical control and corresponding access control. Data link can be divided into dual internal sublayers. Clear sublayer connotation has been protocolled in the fixed index, and the definition can be commonly used. Aiming at the link that industrial control relies on, diversity in communication must be added for consideration of extreme environment. For example, sleep can be added to save energy consumption which fits the new orientation peculiar to real-time energy conservation and communicate various aspects at any time ${ }^{[2]}$.

The third layer is security layer which covers coalition, sets added nodes, and separates the necessary regulations of nodes. Aiming at daily network transmission, it supplies available route. Adding of network security layer maintains the completeness of information and can ensure that the information is complete. This layer has robustness and realizes real-time control of industry. However, the changing internal and external environment as well as diversity should not be neglected. Only in this way can the loads in various time intervals be balanced and can better efficiency be created.

The fourth layer is application layer which integrates much input mapping into network. Multiple refining businesses have been designed on this kind of convergence layer and have been integrated into data flow. Taking the refined network equipment into consideration is convenient for their communication between each other. It supports equipment communication and acquires normal application service. Industrial application fits the regulation under discrete state and also supports continuous control.

\section{Topological property shown by network}

The network of Zigbee has excellent characteristics which can be expanded to tripartite branches namely cluster branch, star-type branch and branch of network type. Various kinds of network all have identifiable and unique transmission advantages, because new-type network under reticular morphology extends necessary transmission paths and the redundant multi-path makes the original network more reliable and simpler. If a path and a node are confronted with breakdown, the fixed path 
will be changed automatically. If confronted with external disturbance, it can also realize automatic switch. Topological structure sets up complicated internal network, extends the general scale shown by nodes, and adds deep route. Topological structure also integrates the self-healing property as well as self-organizing property and decreases the cost fundamentally. Industrial network usually adopts such kind of network.

Network contains internally collocated router, matched terminal equipment and middle coordinator. Available coordinators have been set for coordinating each network. What should be emphasized is that coordinator set as the core maintains the network. Router screens a certain data package in network structure, follows the designed route, and extends the coverage scale. Conversely terminal equipment can be set as unit containing RFD and relevant FFD. Router is connected with coordinator, which is able to simplify existing network equipment and adopt equipment with global functions.

\section{Protocol industrial monitoring should follow}

Industrial control network peculiar to wireless sensor can be convenient for subsequent operation only after setting the protocol and making sure the operation is smooth. Protocol has standards and the control rate is set even lower which is constructed on basis of research and development index. It can be seen from the current situation that protocol is used for broader family security domain, industrial control domain, building control under automatic path, and remote new-type industrial control. In addition, it can also be used for the daily regulation of PC ${ }^{[3]}$.

As for Zigbee, the protocol has presupposed accurate wave band and integrated the spread spectrum and frequency modulation. Protocol constructed in this way will have security and lower overall power consumption. Additional dynamic routing offers networking automatically. ISM can be adopted without application and can be set as frequency band under unified state. In such kind of frequency band, high order modulation has been followed to design the most suitable transfer rate the best of which is 200kbps. Only in this way can more superior general throughput be achieved, can communication latency be narrowed, and can working period be correspondingly reduced. Wireless protocol which is not so complicated supplies the code standard under consistent state.

As for the component category, new-type protocol preserves sufficient internal space which is used for storing transmitted routing information. By promoting the treating performance, protocol has coordination capability. Aiming at internal network, it is convenient for the accessory communication under smooth state and used for topological structure. Functional components integrate multiple prominent advantages such as smaller internal storage, lower device power consumption, etc. It will not coordinate existing network, but can connect with FFD. It can be seen from this perspective that global function component is more similar to existing gateway which contains many multi-nodes in sensors. Such kind of components subtly identifies the new information and transmits it to other gateways.

\section{Design of network architecture}

Network nodes can be divided into conventional nodes and nodes in backbone network. Dual nodes coordinate each other and take responsibility of integrating data and transmitting collected data. Nodes consume relatively more energy, and the periodicity adopts such kind of nodes. Network architecture set by following this kind of thought balances energy consumption and posses balanced advantages.

Nodes have been set for existing equipment of industrial control which automatically constructs sensory networking. Aggregation nodes adopt new monitored data and the PC terminal can distinguish the information and call the police after information inquiry. If a node is damaged, a new node will be added in the device and the route will automatically regulate the networking and add robustness. The sensor is equipped with communication module containing wireless device. Under 
the idle state, the energy consumption between receiving and dispatching is equivalent, which extends the operative year of network and reduces the energy consumption.

For example, a kind of microcontroller manufactured on basis of the technological process peculiar to CMOS contains internal chip and memorizer. Timer adds programmable new characteristics, and joins on-chip simulator. New-type setting, which is more suitable for the sensor network with low energy consumption, can collect accurate information used for subsequent processing. Wireless communication must be equipped with radio frequency module, and load capacitor and crystal oscillator must be added to the chip. Necessary external components are narrowed in order to make sure that the communication path is reliable. Microcontroller also contains received and dispatched wireless module ${ }^{[4]}$.

\section{Real application in industrial control}

\section{Protecting health at any time}

Economy is developing rapidly and faced with increasingly intense competitive pressure. Health degradation gradually becomes a puzzle. Under such kind of situation, real-time device necessary for health inspection must be added, providing normal guardianship. However, some diseases which have gusty representation are hard to be inspected. Potential victim group covers the old, the children and fetus. Taking the medical treatment under shortage state, cover nodes peculiar to Zigbee can be added which will be more suitable for the family small-scale medical treatment. Medical treatment network gradually tends to miniaturization and decreases preferential patient burden. Wireless network protects health and inspects potential disease changes in various periods. The summarized information of guardianship will be integrated to network and share all checkable information.

\section{Fire protection application in plant area}

Fire protection monitoring covers complicated ontology structure and integrates the storage and production workshop in plant areas. Such kind of spatial arrangement is connected with various circuits and extends the general space of fire protection. Wireless monitoring distinguishes multiple terminals and sets matching device of smoke detector ${ }^{[5]}$. For example, smoke detector taken as a kind of necessary terminal collects the smoke. If the smoke with certain concentration is verified, it must be able to call the police immediately. Data management equipped with such kind of center receives real-time alarm which has already been sent, and distinguishes accurate origin of fire, combustion source and potential causes. Setting necessary prevention and control system of fire protection is convenient for dealing with plant area fire disaster under emergency situation.

\section{Combat in new allocation period}

Combat system under new circumstances should be able to consider the man-machine environment under real situations and set refined combat units. Combat system can be integrated into even more complete new-type entirety which integrates equipment, new-type weapon and single pawn. It will be convenient for coordinating action and adds the acquirable effects of combat. Under informatization state, combat system acquires more protruding new advantages. For example, troop soldiers must wear biological nodes peculiar to sensor, and verify and collect changeable vital signs. Necessary device of pulse sensor, device followed by temperature sensor, device adopted by signal amplification, and device of preserved signal can be achieved through imitation. Zigbee regulates the starting and stopping of interface, and screens necessary data format. The satellite system has been set as portable new-type device which can regulate the data format.

\section{Conclusion}

It can be seen from the current investigation and survey situations that wireless sensor network has been used for daily investigation and survey, and become an emphasis point. Zigbee integrates 
various new advantages like lower cost and lower overall power consumption, supporting the necessary wireless network of sensor industrial control. Zigbee has been taken as a sensor technology in newly-developing category which is rooted in existing network. It sets accurate transmission gap, transmission rate, etc. and its normal application is simpler. By creating fictitious scenes, imitating necessary solid model of acquirable monitoring, and verifying various processes of production at any time, new-type network can be set in more fields to highlight the internal value in the future development.

\section{References}

[1] Ji Jinshui. Application of ZigBee wireless sensor network technology in industrial automation monitoring. Industrial Instrumentation \& Automation,2012(03):71-76.

[2] Li Bin, Tian Yaping. Application of ZigBee wireless sensor network technology in industrial fields. Journal of Shanxi University of Science and Technology (Natural Science Edition), 2012(05):110-113.

[3] Yuan Guoliang, Zhong Fei. Application of Zigbee-based wireless sensor network in water level monitoring system. Technical Supervision in Water Resources, 2013(03):31-33.

[4] Zhou Yinan, Zhou Yunsen. Application of WSN wireless sensor network in industrial production real-time monitoring. Industrial Control Computer, 2012(08):24-25.

[5] Lu Nifeng, Wang Peiai. Application of Zigbee-based wireless sensor network in industrial monitoring. Equipment Manufacturing Technology, 2012(04):76-78. 